\title{
«LE MANI LE HO GIALLE PERCHÉ È L'ITALIANO»: BIOGRAFIE LINGUISTICHE E AUTORAPPRESENTAZIONI DI GERMANOFONI IN ALTO ADIGE
}

\author{
Nicole Marinaro ${ }^{1}$
}

\section{INTRODUZIONE}

Diversi autori hanno rilevato la crescente diffusione, nel corso degli ultimi decenni, di studi aventi come oggetto autobiografie linguistiche di parlanti' ${ }^{2}$. Veronesi (2009: 39), ad esempio, osserva che

dagli anni '90 dello scorso secolo in poi si è andata delineando una specifica area di ricerca incentrata sul tema delle «biografie linguistiche» che ha portato a esaminare $i$ percorsi biografici di individui appartenenti a minoranze linguistiche storiche, immersi in situazioni di contatto sullo sfondo di società multilingui o, ancora, essi stessi soggetti di traiettorie di immigrazione.

In tale linea di ricerca si inserisce questo contributo, che si propone di analizzare le rappresentazioni del proprio vissuto linguistico da parte di due gruppi di germanofoni residenti in Alto Adige: persone altoatesine di lingua tedesca, da un lato, e persone provenienti dalla Germania e attualmente residenti in Sudtirolo, dall'altro. Come è noto, un aspetto rilevante della situazione sociolinguistica altoatesina consiste nella diglossia mediale che caratterizza il repertorio del gruppo tedescofono (Vietti, 2008), a sua volta inserito in un contesto di bilinguismo sociale bicomunitario (Mioni, 1990). Data questa premessa, si sono volute indagare le percezioni e i giudizi espressi da persone appartenenti ai due gruppi menzionati rispetto al quadro sociolinguistico in cui sono immersi, nonché rispetto al proprio repertorio e alle proprie esperienze di vita. Questo articolo, in particolare, ha come oggetto la percezione dell'italiano da parte dei due gruppi di informatori.

Il racconto autobiografico degli informatori - 24 in totale, di cui 12 nati in Alto Adige, 11 in Germania e una in Austria - è stato elicitato combinando interviste narrative ${ }^{3}$ e

\footnotetext{
${ }^{1}$ Ulster University.

${ }^{2}$ Come osserva Hu (2006), i contributi caratterizzati da un approccio autobiografico si possono classificare, a grandi linee, in tre diverse categorie: quelli di impronta letteraria, quelli focalizzati sull'aspetto didattico, e quelli dedicati a biografie linguistiche elicitate per mezzo di interviste narrative nel quadro di studi sociolinguistici. Senza alcuna pretesa di esaustività, e premettendo che i diversi campi si possono sovrapporre e influenzare reciprocamente, si rimanda per i primi a Canobbio (2005), Telmon (2006), Groppaldi (2010), Corti (2012). Per gli studi riguardanti l'impiego didattico, si veda, tra gli altri, Adamzik, Roos (2002); Thamin, Simon (2011); i contributi di Cavagnoli e Cognigni in Landolfi (2014); Anfosso, Polimeni, Salvadori (2016); il recente volume curato da Salvadori, Blondeau, Polimeni (2020). Per quanto riguarda, infine, il campo delle scienze del linguaggio, diversi riferimenti bibliografici saranno citati nel corso della trattazione.

3 Rispetto alla tecnica dell'intervista discorsiva semistrutturata si è fatto riferimento principalmente a Cardano (2003).
} 
disegni denominati «(auto)ritratti linguistici», secondo il metodo impiegato da Busch (2006, 2010a, 2010b, 2012, 2013) ${ }^{4}$.

La composizione del campione è sintetizzata nella tabella seguente (Tabella 1):

Tabella 1.

\begin{tabular}{|l|c|c|c|}
\cline { 2 - 4 } \multicolumn{1}{c|}{} & $\begin{array}{c}\text { Provenienza } \\
\text { Alto Adige }\end{array}$ & $\begin{array}{c}\text { Provenienza } \\
\text { Germania }\end{array}$ & $\begin{array}{c}\text { Provenienza } \\
\text { Austria }\end{array}$ \\
\hline $\begin{array}{l}\text { Prima fascia d'età } \\
\text { (18-30 anni) }\end{array}$ & $\mathbf{5}$ & $\mathbf{6}$ & $\mathbf{1}$ \\
\hline di cui maschi: & 3 & 4 & 0 \\
\hline di cui femmine: & 2 & 2 & 1 \\
\hline $\begin{array}{l}\text { Seconda fascia d'età } \\
\text { (45-80 anni) }\end{array}$ & $\mathbf{7}$ & $\mathbf{5}$ & $\mathbf{0}$ \\
\hline di cui maschi: & 4 & 2 & 0 \\
\hline di cui femmine: & 3 & 3 & 0 \\
\hline
\end{tabular}

\section{L'AUTOBIOGRAFIA LINGUISTICA: ASPETTI TEORICO-METODOLOGICI}

Uno dei vantaggi di questo tipo di prospettiva speaker based consiste nel fatto che «l'approccio della biografia linguistica è particolarmente adatto per inoltrarsi in profondità nel vissuto linguistico individuale, nonché per esplorare il rapporto con diverse lingue nel corso della vita» ${ }^{5}$ (Franceschini, 2002: 21). Si tratta, insomma, di assumere una «prospettiva emica che permett[a] di cogliere rappresentazioni e vissuto linguistici per come vengono "offerti" dai parlanti nel quadro del rapporto dialogico instaurato con l'interlocutore nel corso di interviste narrative» (Veronesi, 2009: 54).

Queste parole ci indirizzano verso due concetti chiave emersi da alcune delle principali teorizzazioni elaborate nel campo: da un lato la natura interazionale dei racconti elicitati attraverso interviste narrative, dall'altro gli aspetti sociali e collettivi delle singole biografie.

\subsection{Il racconto autobiografico tra dimensione sociale e interazionale}

In un articolo volto a chiarire il significato del concetto di biografia linguistica, Tophinke (2002) distingue tre accezioni differenti, ma interconnesse, del termine. Nella prima accezione si intende la storia realmente vissuta ("gelebte Geschichte») in relazione all'apprendimento e l'uso delle lingue. Il secondo significato del termine riguarda la ricostruzione mentale dei ricordi («erinnernde Rekonstruktion») di esperienze rilevanti per la propria storia. Infine, il terzo valore di autobiografia linguistica, e l'unico accessibile a un osservatore esterno, è quello di una ricostruzione linguistica («sprachliche Rekonstruktion»)

${ }^{4}$ Si noti, tuttavia, che si è scelto di seguire un ordine inverso rispetto a quello normalmente impiegato da Busch, facendo quindi seguire l'autoritratto linguistico all'intervista narrativa, allo scopo di creare un rapporto di (benché minima) confidenza con l'interlocutore prima di porlo di fronte a un compito che può apparire bizzarro o, per lo meno, poco usuale.

${ }_{5}$ Per ragioni di coerenza interna, si è scelto di tradurre in italiano tutte le citazioni e tutti gli stralci di interviste. Le traduzioni sono di chi scrive. 
del proprio vissuto, realizzata in forma scritta o orale ${ }^{6}$. Oltre a essere legata all'individualità del soggetto che vive, ricorda o racconta le proprie esperienze, ciascuna delle tre accezioni è connotata anche da un aspetto sociale.

Il carattere sociale della gelebte Geschichte è probabilmente il più intuitivo: come è noto, le esperienze linguistiche - o per lo meno quelle legate al parlato - avvengono sempre nella cornice di un contesto comunicativo.

Come si configura, invece, l'aspetto sociale e collettivo delle ricostruzioni mentale e linguistica?

Se, da un lato, la erinnernde Rekonstruktion, in quanto costruzione meramente cognitiva, non è tangibile nel concreto, dall'altro essa è senza dubbio influenzata da schemi di significato sociali. Come spiega Busch (2006: 15), infatti, secondo il concetto di «memoria collettiva» sviluppato da Halbwachs (1997 [1950]) l'inserimento all'interno di un contesto sociale sarebbe cruciale per la costituzione e il mantenimento della memoria individuale, la quale sarebbe influenzata profondamente da condizioni sociali e discorsi dominanti ${ }^{7}$.

Veniamo, ora, alla sprachliche Rekonstruktion: riprendendo Tophinke (2002: 2), l'aspetto sociale deriva, da un lato, dal fatto che la narrazione si svolga sullo sfondo di schemi di significazione collettivi, ai quali si ricorre per conferire senso al proprio racconto; dall'altro lato, la narrazione stessa, in quanto indirizzata a un pubblico - in questo caso, il ricercatore -, consiste a sua volta in un evento comunicativo.

Iniziamo considerando il primo aspetto. Uno dei principali compiti del narratore, come scrive Busch (2006: 14), consiste nella selezione, tra i propri ricordi, delle esperienze ritenute più rilevanti dalla prospettiva attuale. Non si tratta, quindi, della cronaca di una serie di eventi fattuali: ciò che è in atto è un processo di compressione, reinterpretazione e ricostruzione a partire dal (provvisorio) finale della storia (Franceschini, 2001: 114). Tale processo è legato al contesto sociale e collettivo in cui il soggetto si muove (Guerrero, 2011); per questo motivo, negli indirizzi di ricerca biografica in sociolinguistica «la convinzione di fondo è che atteggiamenti e rappresentazioni individuali testimonino modi collettivi e storicamente fondati di dare senso alla realtà (socio)linguistica e abbiano talvolta effetti su di questa» ${ }^{8}$ (Lupica Spagnolo, 2015: 19).

Per quanto riguarda il secondo aspetto collettivo del racconto menzionato da Tophinke (2002: 2), ovvero la natura interazionale e co-costruita dell'intervista narrativa, Veronesi (2019: 2) ribadisce la necessità, articolata da studiosi afferenti a diversi campi del sapere, di non considerare le interviste come un mero strumento per l'elicitazione di dati, ma anche come interazione e pratica sociale?

Nel quadro di una visione ecologica dell'intervista (Berruto, Cerruti, 2014: 217), la contingenza dell'interazione gioca un ruolo cruciale. Riprendendo le parole di Franceschini (2003: 32), «la narrazione viene arrangiata e dettagliata da differenti prospettive in base all'intervistatore e lo scopo, dichiarato e non dichiarato, dell'intervista; così facendo, le unità esperienziali vengono selezionate e delineate». Il concetto fondamentale, insomma, è che «entrambi i partecipanti sono attivamente coinvolti e prendono parte nel costituire la biografia» (Franceschini, 2003: 33). L'intervistato, da un lato, formula ipotesi e supposizioni rispetto all'interlocutore, e, operando un recipient design, adatta la forma e il contenuto della narrazione all'intervistatore (Franceschini, 2003: 3233). Dall'altro lato, anche l'intervistatore influenza attivamente l'interazione attraverso «proposte tematiche, richieste di precisazione e feed-back verbali e non verbali» (Veronesi,

\footnotetext{
${ }^{6}$ Rispetto alle tipologie di informazioni o 'realtà' ottenibili attraverso racconti biografico-narrativi, si veda anche Nekvapil (2003), Pavlenko (2007), Cognigni (2020).

${ }^{7}$ A proposito di memoria e ricordo, in particolare in riferimento alla scrittura autobiografica, si veda anche Blondeau, Salvadori (2020: 20-22).

${ }^{8}$ Cfr. anche Nekvapil (2003), Fix (2003), Franceschini (2004) e Franceschini, Miecznikowski (2004).

${ }^{9}$ Cfr. anche Pavlenko (2007).
} 
2008: 124); per quanto possa assumere un atteggiamento non impositivo, al ricercatore continua infatti a spettare il ruolo di regista dell'intervista.

Come illustrato dallo schema riportato poco sotto (Figura 1), Franceschini (2003: 32$33)^{10}$, distingue una dimensione dell'interazione («interactional dimension») e una dimensione della presentazione («presentational dimension»). La dimensione interazionale si colloca nel bic et nunc (momento $\mathrm{T}$ ) in cui la biografia viene raccontata, ma gli eventi e le esperienze cui il racconto si riferisce, e che vengono paragonati a eventi ed esperienze del presente, sono legati a un momento del passato $\mathrm{T}-1$. La dimensione presentazionale, invece, permette da un lato di trarre inferenze riguardo a eventi, attitudini e circostanze concernenti il narratore, dall'altro di risalire a riferimenti storico-collettivi, quali avvenimenti politici, migrazioni di massa o guerre.

La dimensione presentazionale e la dimensione interazionale sono strettamente legate: come illustra l'immagine, la biografia emerge, in un movimento a spirale, da un processo circolare in cui la contingenza dell'interazione si fonde con i riferimenti relativi al passato. «La biografia linguistica [...] si configura dunque come evento comunicativo in cui quattro poli - narratore, ascoltatore, autobiografia e storia collettiva - si intrecciano ed interagiscono tanto sul piano dialogico-interazionale quanto su quello presentativo» Veronesi (2008: 124).

Figura 1.

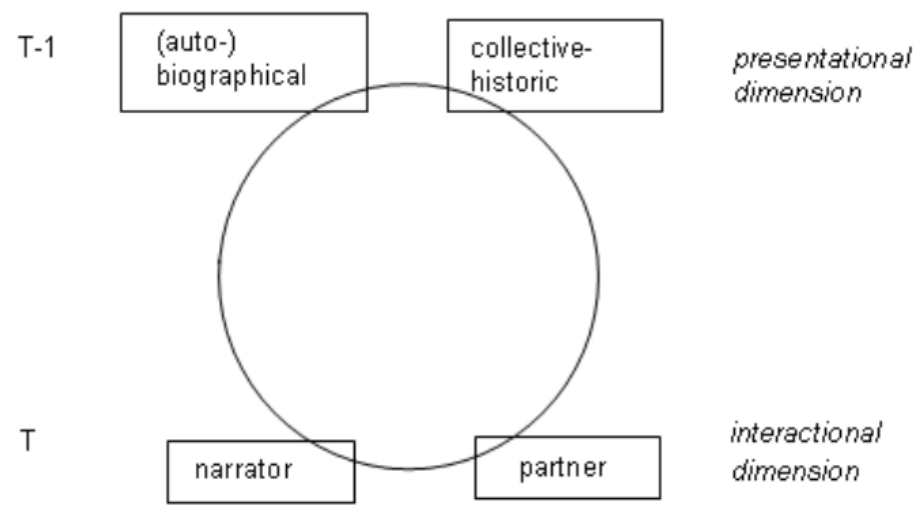

Quanto detto finora non vale, naturalmente, solo per l'intervista qualitativa, ma anche per l'autoritratto linguistico, sul quale si tornerà in seguito.

Per quanto riguarda l'effetto dell'interazione contingente sui dati raccolti, nella presente indagine gli elementi che potrebbero aver maggiormente influenzato $i$ racconti autobiografici sono la modalità di svolgimento dell'intervista (di persona o online) ${ }^{11}$, la lingua impiegata (italiano o tedesco) ${ }^{12}$, e, infine la familiarità degli informatori con il background dell'intervistatrice.

Alle considerazioni delineate finora si lega l'aspetto metodologico forse più delicato nell'ambito degli studi basati su percezioni e autorappresentazioni, ovvero

${ }^{10}$ Cfr. anche Franceschini (2001: 115).

11 A causa della lontananza fisica di alcuni informatori e del riacuirsi della pandemia nella fase finale della raccolta dei dati (autunno 2020), 9 delle interviste si sono svolte a distanza. Una delle maggiori difficoltà connesse a questa modalità ha riguardato certamente la realizzazione dell'autoritratto linguistico da parte degli intervistati.

12 Per privilegiare la naturalezza dell'interazione, alcune delle interviste condotte con informatori altoatesini (in totale 5) si sono infatti svolte in italiano. Non verrà qui approfondita, invece, la questione della differenza tra la varietà di tedesco parlata dall'intervistatrice e quella parlata dagli informatori altoatesini. 
l'interpretazione dei dati ottenuti (Berruto, Cerruti, 2014: 217). Diversi autori hanno rilevato la divergenza fra le valutazioni esplicite e il comportamento reale degli informatori, riconducibile principalmente a due fattori: da un lato, il carattere irriflesso della maggior parte dei comportamenti linguistici, dei quali spesso il parlante ha «scarsa o nulla consapevolezza» (Dal Negro, 2012) ${ }^{13}$; dall'altro, «le risposte possono essere improntate a, o condizionate, da atteggiamenti ideologici e proiezioni (non necessariamente consapevoli) di comportamenti preferiti, o attesi, o voluti, per una ragione o per l'altra, sui comportamenti reali» (Berruto, Cerruti, 2014: 218). L'informatore, cioè, tende a voler trasmettere un'immagine di sé il più possibile positiva, che «corrisponda alle (presunte) aspettative del ricercatore» (Iannàccaro, 2002: 86), oppure che «vada ad avvalorare un'idea o ideologia condivisa» (Dal Negro, 2012: 23) ${ }^{14}$.

\subsection{L'autoritratto linguistico: disegnare il proprio repertorio}

L'autoritratto linguistico è uno strumento didattico e di ricerca che permette una rappresentazione creativa del repertorio linguistico del soggetto, particolarmente utile per l'esplorazione di prospettive emiche e soggettive in contesti di plurilinguismo ${ }^{15}$.

Come spiega Busch (2018), il modo di rappresentazione grafico segue una logica diversa rispetto a quello verbale, e permette di spostare il fuoco dell'attenzione rispetto a una narrazione orale. Mentre lo scritto e il parlato sono determinati dal tempo e da una sequenzialità lineare, l'immagine è caratterizzata dallo spazio e dalla simultaneità; il racconto orale necessita di continuità diacronica e coerenza sincronica, mentre nel disegno possono coesistere contraddizioni, rotture, sovrapposizioni e ambiguità. La rappresentazione visiva indirizza l'attenzione sull'intero, sulle proporzioni e sulle relazioni reciproche tra le singole parti, offrendo molteplici possibilità di espressione attraverso la posizione, forma e dimensione dei campi colorati, il colore stesso, l'intensità e la saturazione delle tonalità; anche le aree lasciate bianche assumono frequentemente un significato specifico. Disegnare, inoltre, permette di fermarsi, riflettere e prendere distanza, sfuggendo alla 'costrizione ad agire' e alle razionalizzazioni affrettate che può comportare la narrazione.

Il pensare e rappresentare attraverso immagini costituisce quindi una modalità autonoma della creazione di senso, che struttura il racconto e permette di far emergere aspetti ulteriori rispetto alla sola intervista. Le spiegazioni degli informatori, elicitate a partire dall'immagine, ruotano spesso intorno a metafore legate al colore e al corpo umano: la testa viene spesso concettualizzata come la sede della ragione, ma anche dello sforzo cognitivo; il cuore è collegato agli affetti e alla familiarità, le gambe alla perifericità, $\mathrm{ma}$ in altri casi anche al sentirsi radicati in un luogo o in una lingua. L'utilizzo del colore, a sua volta, può essere legato a connotazioni diffuse, quali il rosso per le emozioni o il blu per la distanza, ma anche a preferenze e associazioni personali. Sono frequentemente impiegate metafore spaziali, quali dentro/fuori, per esprimere una sensazione, rispettivamente, di appartenenza o estraneità, o grande/piccolo, per una maggiore o minore importanza, nonché elementi iconici e simbolici, quali le bandiere nazionali.

È importante tenere in considerazione che le singole rappresentazioni, pur ispirandosi spesso a schemi simili, differiscono ampiamente rispetto al modo in cui i partecipanti

${ }^{13}$ Cfr. anche Dell'Aquila, Iannàccaro (2000).

${ }^{14}$ Cfr. anche Iannàccaro (2000) e Iannàccaro, Dell'Aquila (2006).

15 Per un'ampia rassegna sull'utilizzo del medium visivo nella ricerca sul plurilinguismo si rimanda a Kalaja, Melo-Pfeifer (2019); si veda anche Molinié (2009). 
assegnano (o meno) particolari significati ai singoli elementi del disegno. È necessario, quindi, evitare oggettivazioni avventate: il significato del ritratto non può essere assunto a priori da un osservatore esterno, ma diviene chiaro solo nel momento in cui viene illustrato dall'autore stesso. Il disegno non può, quindi, essere considerato quale rappresentazione criptata di una verità inaccessibile alla consapevolezza; come la narrazione orale, l'autoritratto e la spiegazione a esso legata sono radicati nella contingenza dell'interazione tra partecipante e intervistatore. Castellotti e Moore (2009) osservano che le rappresentazioni non possono essere ritenute giuste o sbagliate, né permanenti: esse, piuttosto, permettono all'informatore di decidere quali siano le caratteristiche rilevanti nella costruzione della propria identità.

Busch (2018: 60) invita poi a tenere in considerazione come, oltre agli elementi che influenzano qualsiasi tipo di rilevazione qualitativa, nel caso del ritratto linguistico assumano grande importanza anche il tipo di sagoma utilizzato e la formulazione delle istruzioni. Per quanto riguarda la silhouette da fornire agli informatori, nel presente studio, seguendo hahaha (2018), si è deciso di adottare quella sviluppata secondo i criteri dell'arteterapia dalla stessa studiosa (Figura 2$)^{16}$.

Figura 2.

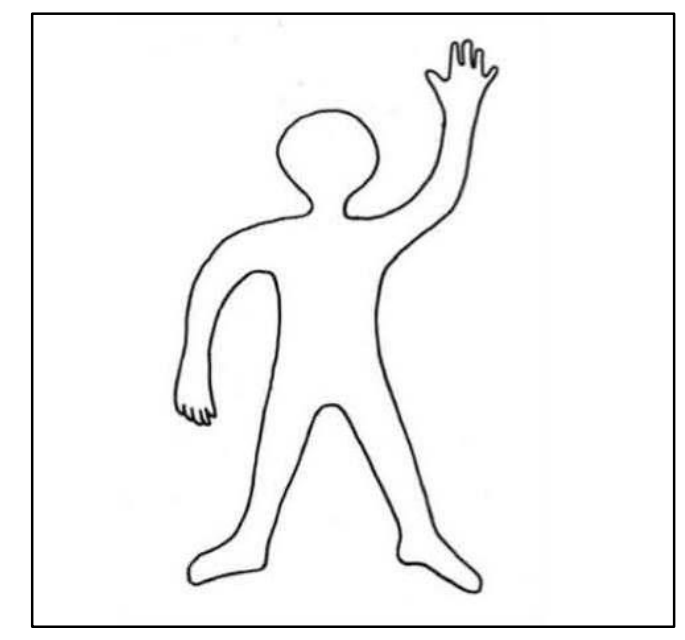

\section{ANALISI DEI DATI}

\subsection{Percezione dell'italiano da parte degli informatori germanici}

Il rapporto con l'italiano rappresenta un tema di grande importanza nella narrazione degli informatori germanici; in effetti, a differenza di quanto accada per il dialetto sudtirolese, tale codice trova uno spazio più o meno esteso nell'autoritratto linguistico di tutti i partecipanti. In generale, si può affermare che le valutazioni espresse riguardo allitaliano siano positive, mentre varia, in base alle singole esperienze e traiettorie di vita, il livello di competenza degli informatori e la rilevanza assunta dalla lingua nella quotidianità.

16 Disponibile all'indirizzo http://heteroglossia.net/Sprachportraet.123.0.html. Per la motivazione della scelta di questo tipo di sagoma e per la formulazione delle istruzioni fornite agli informatori, si rimanda a Busch (2018: 60-63; 2021: 9). 
In molti casi, la domanda dell'intervistatrice rispetto alla relazione dell'interlocutore con l'italiano elicita un racconto, più o meno dettagliato, riguardo il processo di apprendimento della lingua.

Nel caso dell'informatrice Pa.de $2^{17}$, arrivata in Alto Adige all'età di cinque anni, il rapporto positivo con questo codice è favorito dal forte contrasto rispetto all'esperienza traumatica dell'apprendimento del dialetto locale. Mentre l'incontro-scontro con il Südtiroler Dialekt è connotato come esperienza di esclusione da parte del peer group, quello dell'acquisizione dell'italiano è legato invece all'atmosfera giocosa delle amicizie in cortile. Nel corso dei decenni, l'italiano è poi divenuto una parte importante della comunicazione quotidiana dell'informatrice: ciò risulta evidente dallo spazio dedicatogli nel suo ritratto (in azzurro), nonché dal significato metaforico delle parti del corpo scelte (Figura 3).

L'altra gamba ${ }^{18}$ con cui sto nel mondo è l'italiano, faccio molto con l'italiano (. $)^{19}$ cioè quello che faccio, quello con cui mi occupo, lo disegno nel braccio e nella mano [...] Metà della mia testa è italiana, metà della mia testa è in qualche modo occupata con l'italiano. Penso anche in italiano, parlo in italiano.

Figura 3.

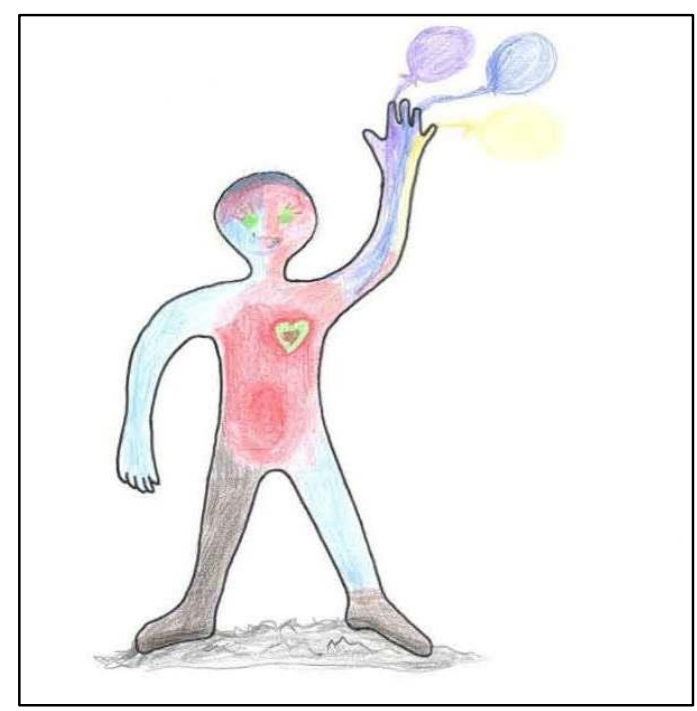

Anche per l'informatrice Bi.de2, residente a Bolzano da circa 30 anni, l'italiano sentito fin dall'infanzia durante le vacanze trascorse in Alto Adige con la famiglia - è divenuto un elemento costitutivo del repertorio. L'informatrice racconta dettagliatamente il processo di apprendimento della lingua, dall'esperienza da au pair all'incontro con il

${ }^{17}$ Per facilitare l'associazione alla provenienza geografica e all'età anagrafica, la sigla con cui si indicheranno gli informatori sarà composta nel seguente modo: prime due lettere del nome puntate, abbreviazione indicante la provenienza geografica ("st" per gli altoatesini, "de" per i germanici, "au" per l'informatrice austriaca), numero indicante la fascia di età (1 per la fascia d'età tra i 18 e i 30 anni, 2 per la fascia d'età tra i 45 e gli 80).

18 Nella prima gamba, dopo una lunga esitazione, l'informatrice aveva invece rappresentato il dialetto sudtirolese, in virtù della prominenza di tale codice nella sua vita quotidiana.

${ }^{19}$ Le convenzioni di trascrizione adottate, che seguono in buona parte quelle impiegate da Veronesi (2009), sono le seguenti: il punto indica intonazione finale di scendente, la virgola intonazione sospensiva o lievemente ascendente, uno o più punti tra parentesi pausa più $\mathrm{o}$ meno breve; il trattino indica una riformulazione; le risate sono segnalate con «[ride]»; i puntini di sospensione tra parentesi quadre indicano l'omissione di parti dell'estratto. Quando riportati, i turni dell'intervistatrice sono indicati con "N.". 
futuro marito, bolzanino bilingue, fino agli studi universitari, compiuti a Verona e a Trento, che la portano a perfezionarne la conoscenza. Anche qui, l'importanza dell'italiano è rispecchiata dalle scelte grafiche dell'informatrice (Figura 4): a questa lingua, infatti, assegna il tronco, a rappresentare un elemento centrale della sua vita, e sceglie il colore blu,

il colore dell'acqua [...] nel corso del tempo l'elemento 'acqua' è diventato la base dei miei hobby preferiti, a seconda della stagione e in molte sfaccettature.

Al contempo, attraverso gli spazi bianchi, nel tronco viene anche rappresentato il Südtiroler Dialekt, un codice che l'informatrice dichiara di non aver mai voluto utilizzare in quanto estraneo alla propria identità, ma parlato quotidianamente dai figli.

Figura 4.

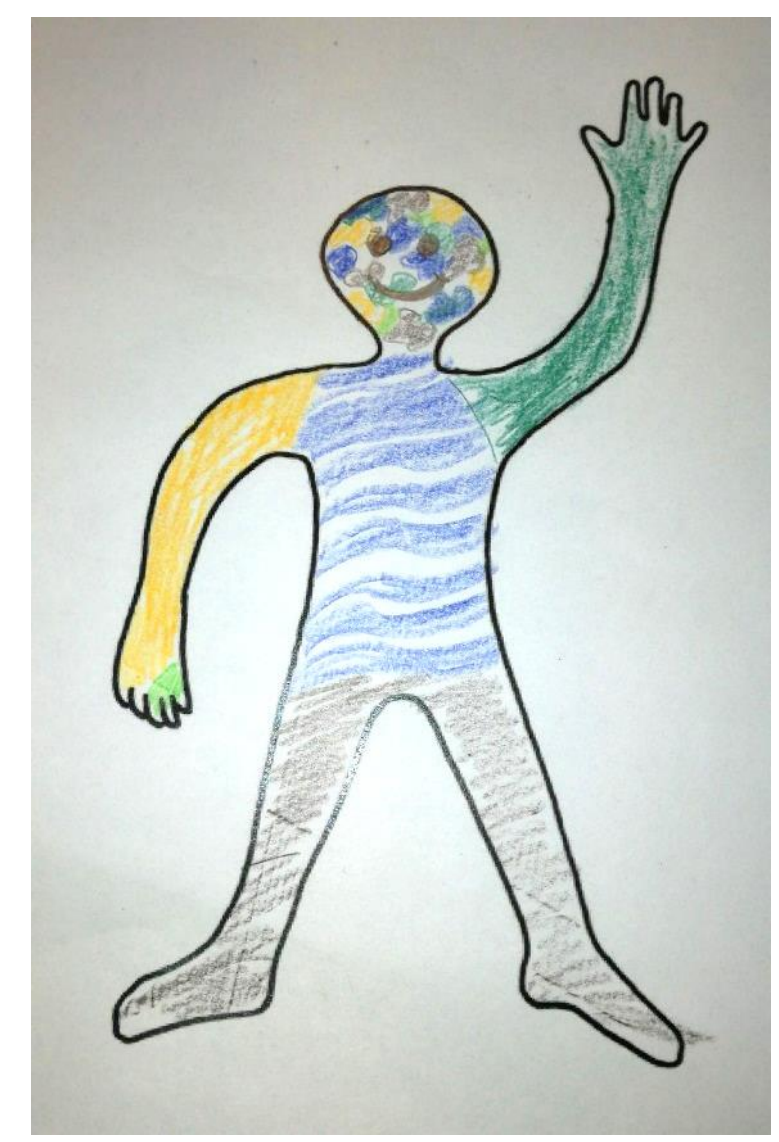

Il periodo universitario è rappresentato come un momento fondamentale per l'apprendimento dell'italiano anche nel racconto di Di.de2, che ricorda come, grazie alla frequenza di un corso intensivo di lingua, si era poi potuto iscrivere alla facoltà di veterinaria presso l'università di Pavia. Dal ritratto dell'informatore (Figura 5) emerge una visione primariamente strumentale della lingua, la quale, parimenti al dialetto sudtirolese, viene concettualizzata nel quadro dell'utilità ai fini del proprio mestiere: la loro posizione nelle braccia e nelle mani (in azzurro l'italiano, in verde il dialetto) è legata al fatto che corrispondano alle lingue della quotidianità lavorativa. 
Figura 5.

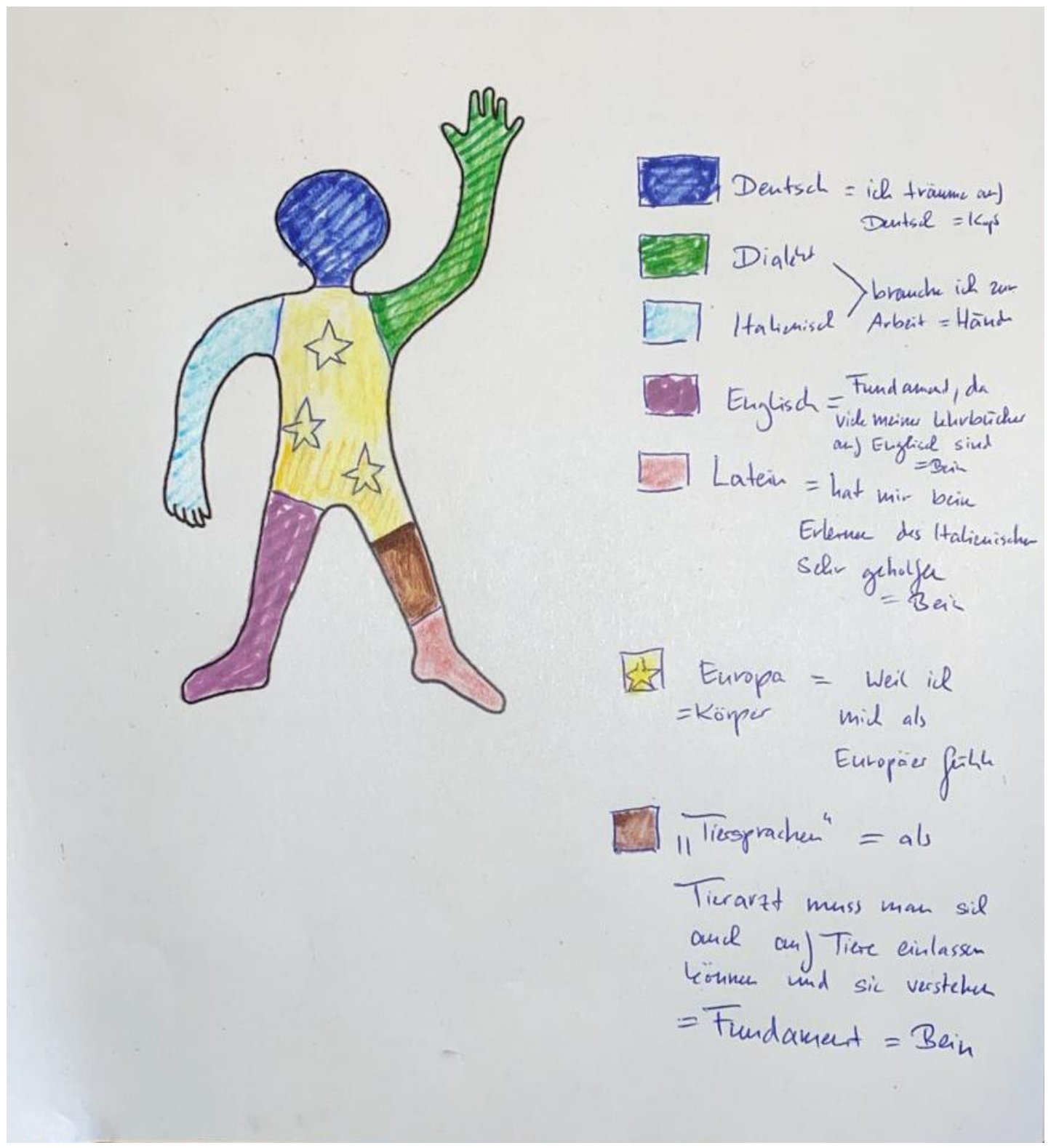

Nel caso di Re.de2, medico e fratello di Di.de2, l'apprendimento viene inizialmente vissuto come una costrizione, necessaria per conseguire la certificazione di bilinguismo richiesta per esercitare la professione in ambito pubblico; la vergogna per la scarsa competenza induce l'informatore a evitare il più possibile l'italiano, fino a che l'incontro con la futura moglie, italofona, lo aiuta a liberarsi da quest'inibizione. Come illustrato nel suo autoritratto linguistico (Figura 6), nell'attuale quotidianità dell'informatore l'italiano (in blu) ricopre circa la metà della comunicazione, e rappresenta la lingua straniera meglio padroneggiata. 
Figura 6.

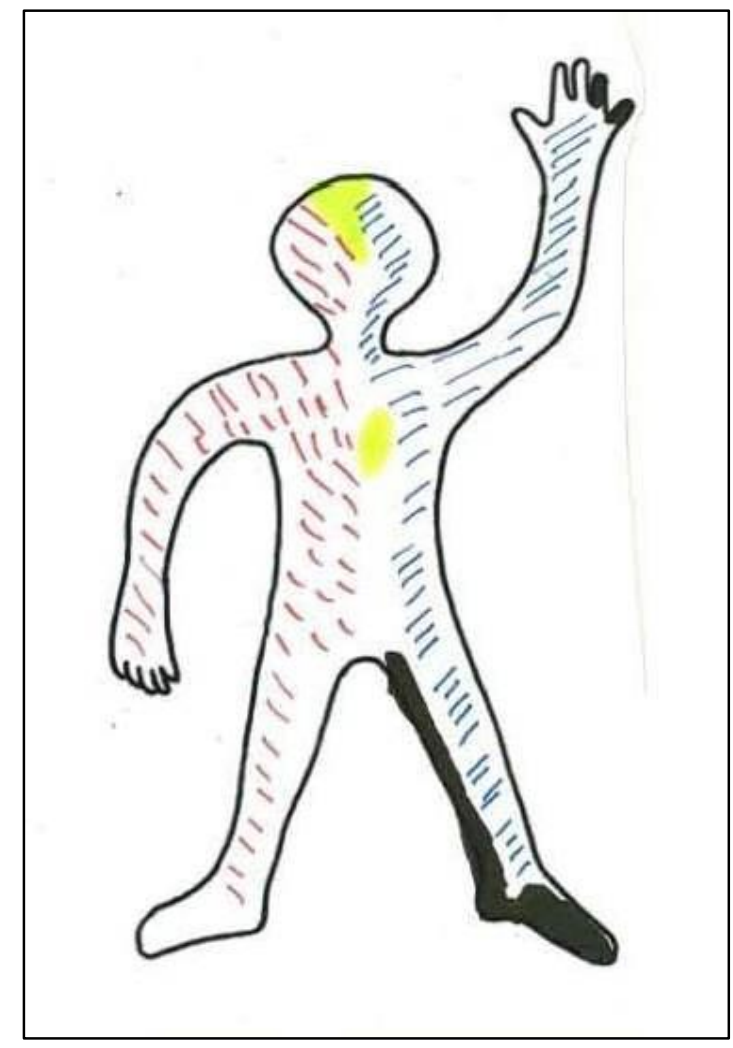

Parzialmente simile è il racconto di Br.de2, trasferitasi a Bolzano da giovane, e che sottolinea l'importanza della padronanza dell'italiano per comunicare con i clienti della farmacia in cui lavorava. L'informatrice ricorda che, nel quadro della temperie degli scontri tra gruppi etnici degli anni '60, alcuni altoatesini di lingua tedesca la scoraggiavano dall'imparare l'italiano: «Non serve che impari l'italiano, che imparino il tedesco i $W$ alschen [=termine dispregiativo per gli italiani]». L'informatrice, tuttavia, rievoca la propria determinazione nell'apprendere la lingua, in virtù della fondamentale importanza della chiarezza della comunicazione in ambito medico:

Al lavoro - al lavoro iniziai a tenere un quaderno e ci scrivevo tutto e lo studiavo la sera in modo da sapere le cose per la volta successiva. [...] Annotavo diligentemente sul mio quaderno e poi ripetevo e ripetevo e brigavo e facevo ed è così che ho imparato ${ }^{20}$.

L'informatrice ricorda poi come momento saliente nel suo processo di apprendimento dell'italiano la frequenza di un corso di lingua incentrato principalmente sull'aspetto culturale: in particolare, grazie a un viaggio durante il quale visita diverse città d'Italia, la percezione dell'informatrice cambia significativamente.

Lì poi vedi l'italiano in modo diverso, non lo vedi come una lingua straniera, in modo così teorico, ma capisci qualcosa dalle emozioni [...] Se si ha

20 Tanto Br.de2 quanto Bi.de2 impiegano, nella propria narrazione, quella che Ellis (2001) definisce la metafora dell'apprendente come problem-solver e worker. 
un'avversione interiore, «questo è il mio avversario», allora non lo imparo. Se non ci si interessa, se non si ha nessun contatto con la storia degli italiani, allora rimarrà sempre una cosa teorica, lo faccio solo perché devo farlo e altrimenti - e così era un'altra cosa, era così bello, mi piaceva davvero tanto, e anche imparare diventa più facile.

Nel proprio ritratto linguistico (Figura 7), l'informatrice rappresenta quindi l'italiano con il colore giallo, che lega a impressioni positive.

Figura 7.

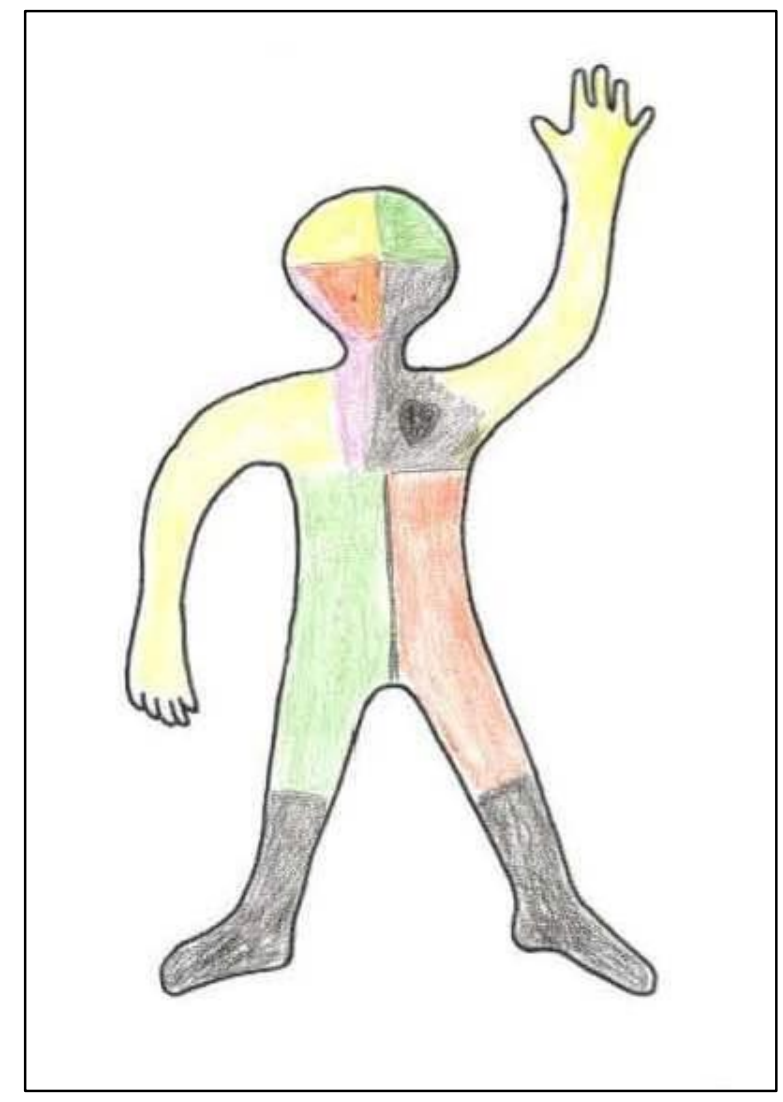

La scelta del colore giallo è comune anche all'informatrice Me.de1, la quale racconta di parlare molto l'italiano, soprattutto con persone conosciute nell'ambito dello sport, e di averne una buona competenza ${ }^{21}$. Pur conscia di essere, per lo meno parzialmente, influenzata da luoghi comuni, non può fare a meno di ritenere l'italiano «una lingua romantica» e con «un carattere forte»; nel proprio ritratto (Figura 8), sceglie di rappresentarlo nelle mani.

Le mani le ho gialle perché è l'italiano, perché si gesticola molto - perché si gesticola tutto il tempo e anche per comunicare un po' meglio uso anche le mani quando lo parlo, ed è giallo perché per me è una lingua molto calda e per me è un po' collegato con la temperatura calda, ma anche con le vacanze estive.

21 Competenza che, in effetti, viene dimostrata durante l'intervista dal disinvolto code-switching dell'informatrice. 
Figura 8.

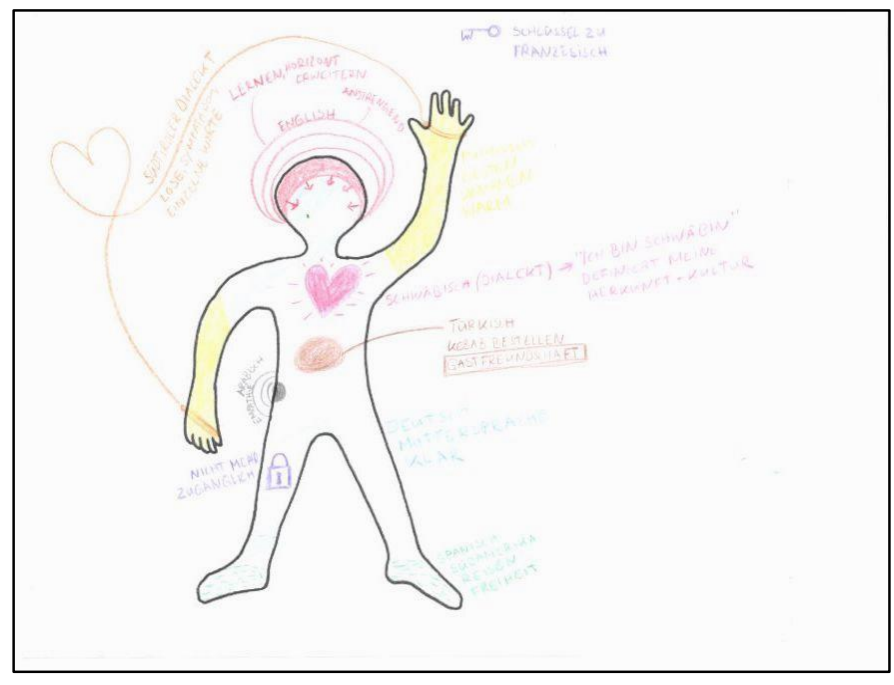

L'elemento delle mani ritorna in diversi autoritratti: come Me.de1, anche l'informatrice Em.de1, che concettualizza l'italiano attraverso tre metafore legate al corpo, fa riferimento ai gesti (Figura 9).

L'italiano [...] una qualche tonalità di rosa [...] l'italiano credo che nel mio caso abbia luogo molto nella testa perché ci penso molto e parlare italiano non mi risulta necessariamente intuitivo, cioè non in ogni situazione e rispondo anche forse in modo cerebrale, non proprio così liberamente, non scorre o così (.) e direi in ogni caso che le mani sono italiane per me (..) perché in Germania non parlavo così tanto con le mani ma in Italia ha un significato completamente diverso e che ci sono gesti che prima non conoscevo e che qui hanno un significato molto speciale, e devo - vorrei imparare a leggere le mani per capire le persone [...] ma l'italiano corrisponde alle mani anche non solo perché è importante per gli italiani e le italiane ma anche quando voglio comunicare nella vita quotidiana e non so qualcosa, allora devo spiegare molto con le mani in qualche modo. [...]

Forse faccio anche il mio cuore un po' italiano perché spero che un giorno la potrò considerare come lingua del cuore, un giorno.

Figura 9.

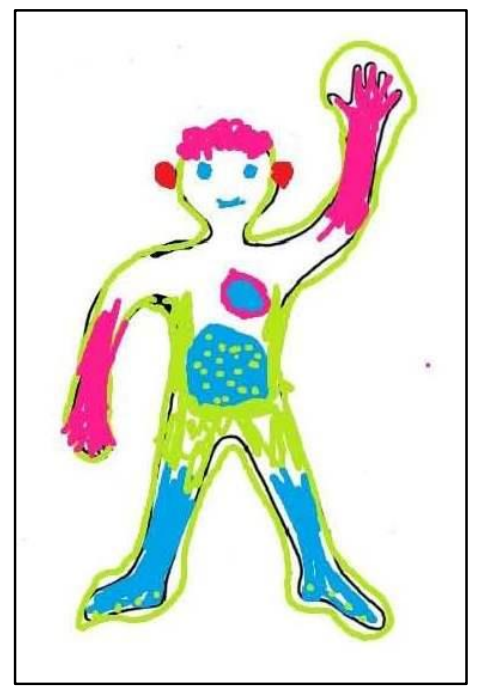


Le.au1, invece, che al momento dell'intervista stava seguendo un corso intensivo di italiano, rappresenta attraverso le mani il proprio processo di apprendimento (Figura 10):

Il rosso è l'italiano, lo metto nelle mie mani perché devo ancora scrivere molto, imparare molto, scrivere le cose, annotarmi le cose.

Figura 10.

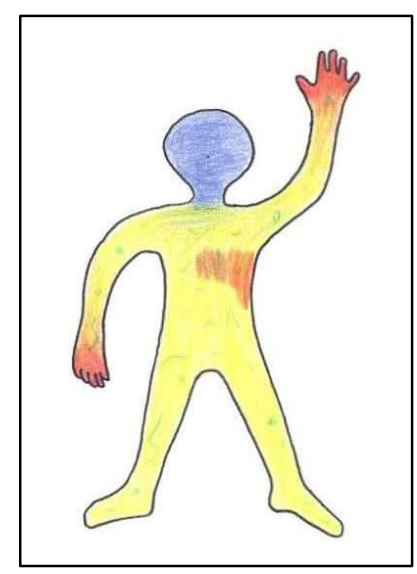

In altri ritratti predomina invece la rappresentazione, in un certo senso statica, di una competenza linguistica imperfetta. Ad esempio, nel ritratto dell'informatore Re.de1 l'italiano (in verde) viene raffigurato come una delle lingue nelle quali l'informatore si sente meno sicuro, ma, al contempo, come una di quelle che più ama parlare (Figura 11).

La non perfetta competenza in italiano, come anche quella in altre lingue, viene inoltre rappresentata come uno scudo che protegge il parlante dal bisogno di esprimersi con precisione estrema. Per portare un esempio, Re.de1 commenta:

Effettivamente ho meno esitazioni a fare una domanda a un docente italiano che a qualcuno in inglese, perché in inglese [lingua di cui ha un'alta competenza] sentirei il bisogno di esprimermi in modo ultra preciso.

Figura 11.

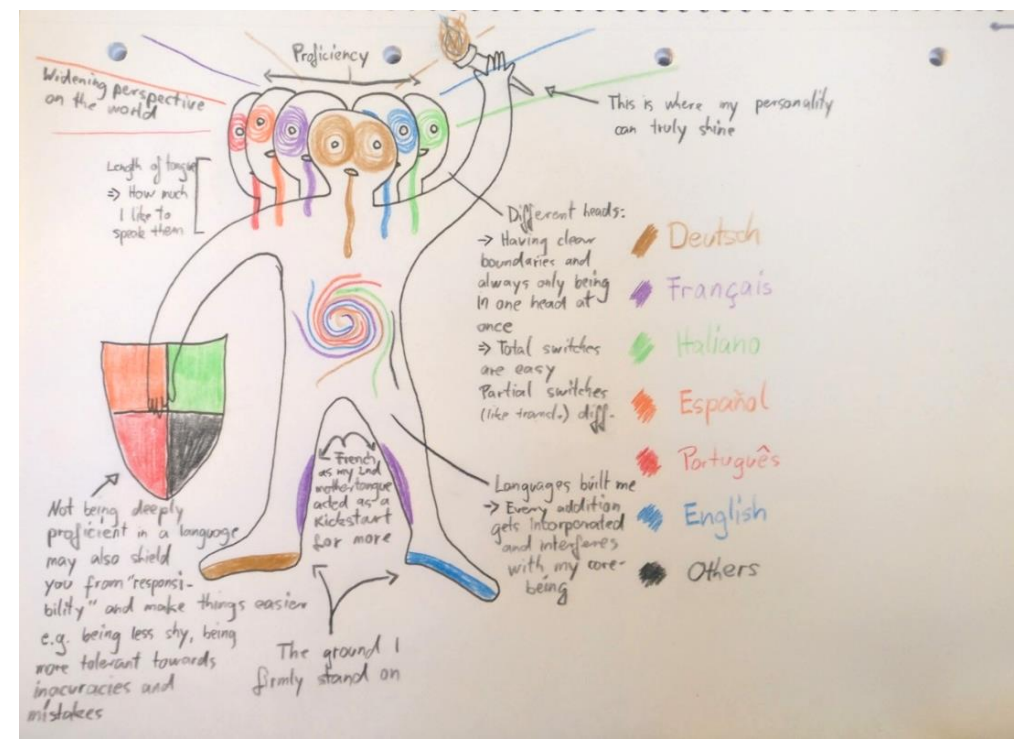


Ti.de1, nonostante la buona competenza linguistica, e pur dichiarando di apprezzare molto la possibilità, all'interno del suo cerchio sociale, di passare da una lingua all'altra senza alcun problema, esita lungamente al momento di scegliere come rappresentare l'italiano, ed esterna un lieve disagio a riguardo:

Non so bene che posto abbia l'italiano dentro di me. Il fatto è che non mi sento davvero - sento il mio accento tedesco quando parlo italiano anche quando - perché la $\mathrm{R}$ non la so pronunciare per esempio [...] non mi sento davvero a casa nella lingua. Penso che sia una lingua super super bella, ma quando la parlo, in qualche modo non riesco a sentirmici inserito dentro.

Dopo una lunga riflessione, l'informatore decide di rappresentare l'italiano (in viola) nella pancia (Figura 12); la motivazione, però, è opposta rispetto a quanto accade nel caso di altri ritratti, in cui la lingua rappresentata nella pancia è quella percepita come più facile e spontanea.

Non lo so, penso che l'italiano sia nel mio stomaco perché in qualche modo - in qualche modo non è da nessuna parte nel mio corpo o nel mio cuore o nella mia testa, ma è nel mio stomaco, è sempre lì e quando ne ho bisogno allora posso tirarlo fuori.

Figura 12.

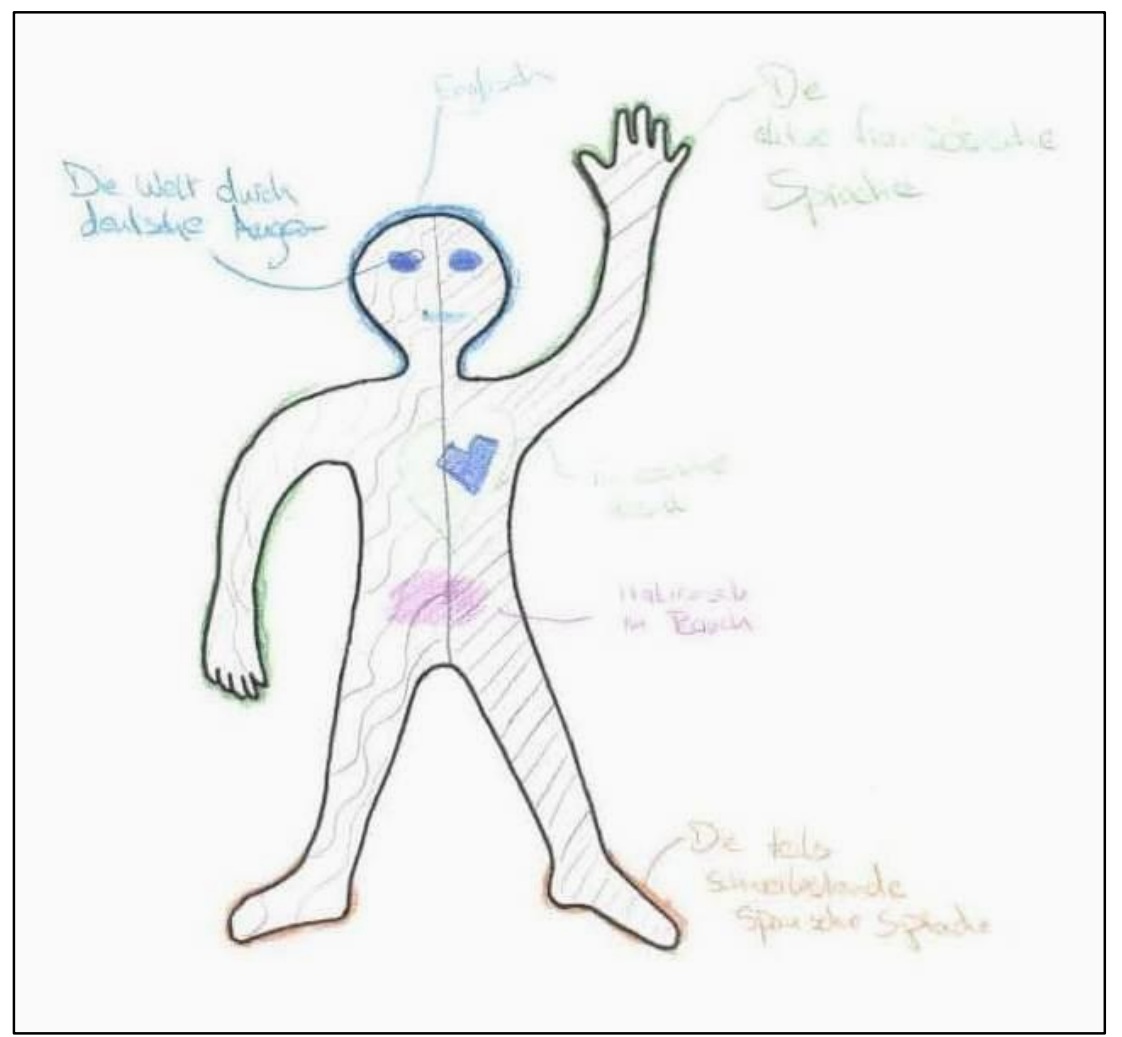

Anche Ko.de1 sceglie una posizione concettualizzata come periferica, ovvero il braccio, per rappresentare la sua debole competenza in italiano. In questo caso, secondo uno schema piuttosto ricorrente, l'informatore utilizza la bandiera nazionale per indicare la lingua (Figura 13). 
Figura 13.

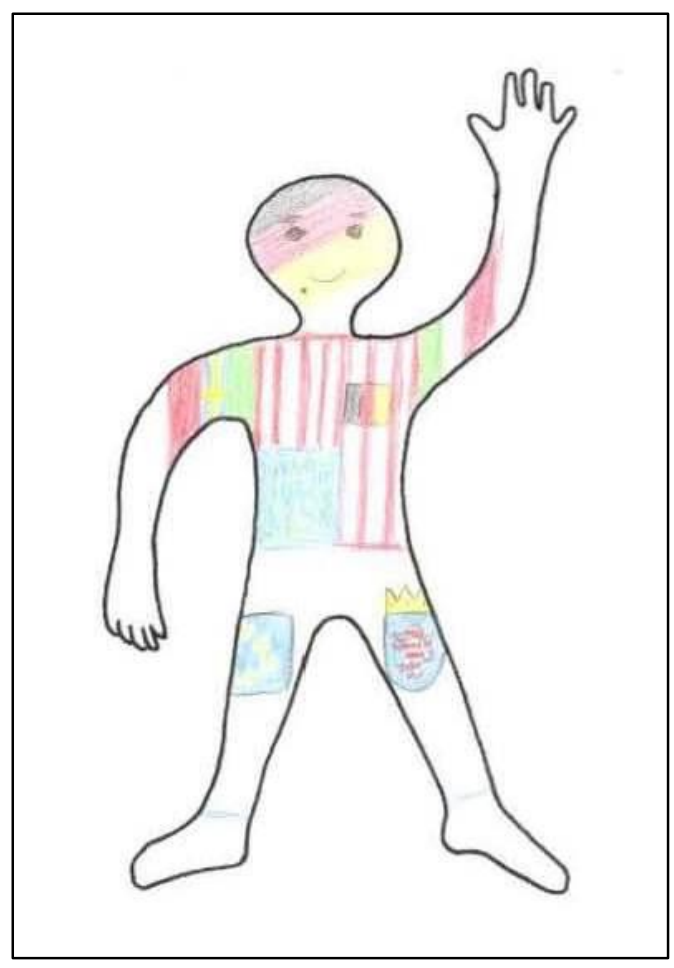

Mo.de1, infine, che dichiara di capire bene l'italiano, ma di avere remore a partecipare attivamente a una conversazione, rappresenta il ruolo non ancora centrale di questo codice attraverso una sagoma di dimensioni ridotte, in verde (Figura 14).

Figura 14.

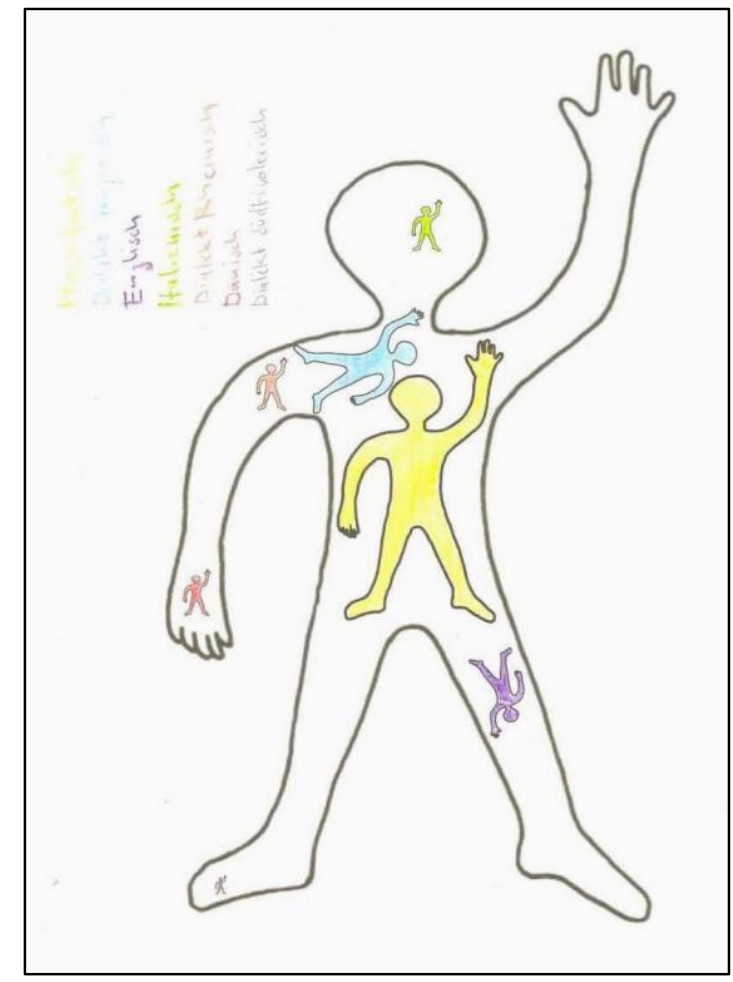




\subsection{Percezione dell'italiano da parte degli informatori altoatesini}

Venendo ora agli informatori altoatesini inclusi nel campione, tutti gli intervistati si considerano bilingui, per quanto a livelli di competenza diversi. Non stupisce, quindi, che l'italiano giochi un ruolo prominente nei loro racconti; è invece interessante notare che, come già osservato per gli informatori germanici, esso sia spesso presentato come più saliente rispetto a quello del dialetto ${ }^{22}$. La percezione dell'importanza dell'italiano nella vita degli informatori è rispecchiata anche dallo spazio dedicatogli nei ritratti linguistici.

Diversi informatori, specialmente se provenienti da famiglie bilingui, rappresentano graficamente una mescolanza tra i due codici: ne è un esempio l'autoritratto linguistico di Ph.de1 (Figura 15), che sceglie colori simili tra loro - rispettivamente verde acqua e blu per segnalare il fatto che, nella sua percezione, italiano e tedesco «sono la stessa cosa, rappresentano la stessa cosa». Le due lingue si posizionano infatti in parti eguali nel cuore dell'informatore e partecipano allo stesso modo tanto della struttura più solida della figura, nelle gambe, quanto, in «un passaggio fluido, spontaneo», del suo «vivere e percepire»; infine, sono entrambe soggette a un breve momento di riadattamento quando, dopo un periodo passato a parlare una delle due lingue, Ph.st1 deve tornare a utilizzare l'altra.

Figura 15.

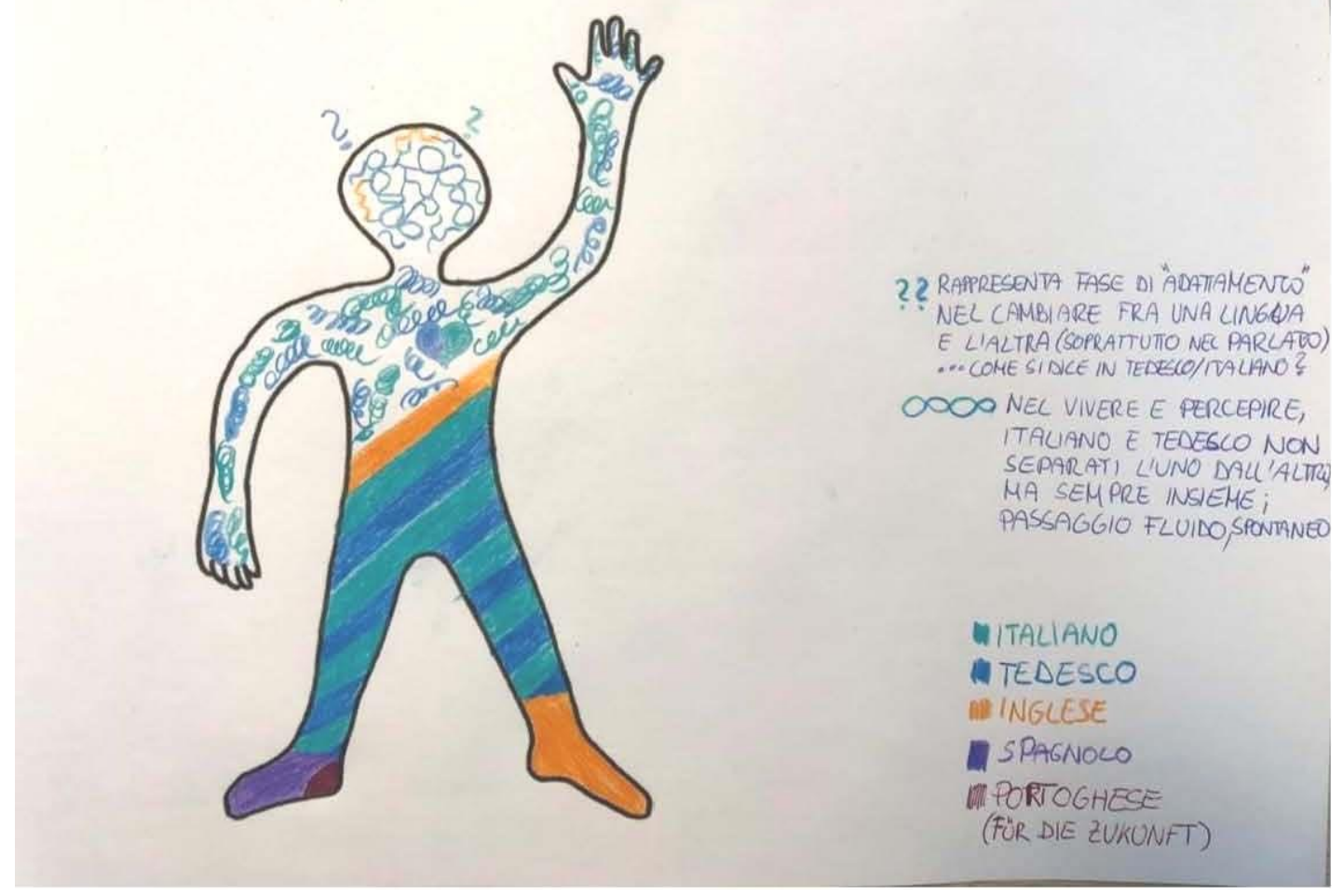

22 In molti casi, la domanda dell'intervistatrice rispetto alla lingua percepita come propria lingua madre elicita una risposta che si configura come risultato della scelta tra italiano e tedesco; solo in seguito a una richiesta di chiarimento ulteriore gli informatori specificano se intendessero il dialetto o la Hochsprache. Una possibile spiegazione potrebbe derivare dal fatto che molti informatori diano per scontato che si intenda il dialetto. 
Anche Aa.st1 - a sua volta proveniente da una famiglia bilingue - rappresenta quale elemento chiave del proprio ritratto la mescolanza dei due codici, senza però assegnare i colori (giallo e blu) alle rispettive lingue (Figura 16):

Mi è venuto non so perché di disegnare una spirale, tipo tedesco italiano, perché comunque è tutto un miscuglio (.) cioè non - tipo per me tipo anche lo stesso livello di lingua, italiano tedesco fanno entrambe parte di me e quindi le mescolo le - Cioè quindi non mi viene nemmeno di disegnare una parte così e una parte così ma tipo una roba - tutta insieme.

Figura 16.

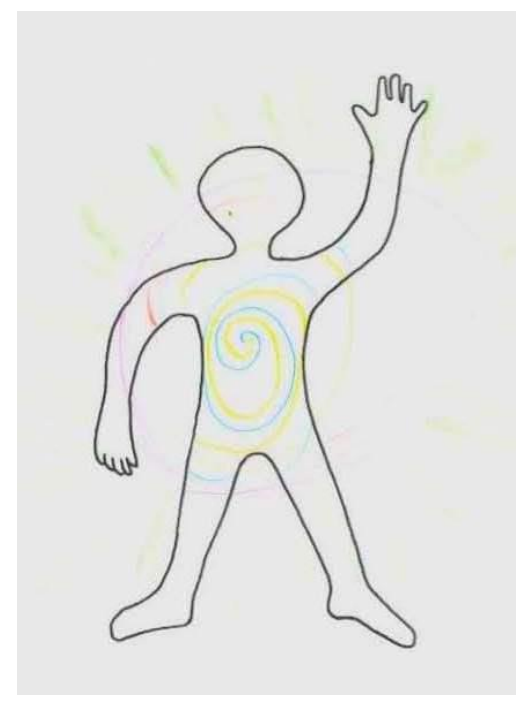

Viene assegnato a entrambe le lingue un ruolo equivalente anche dall'informatrice Ev.st2, che, pur di famiglia esclusivamente tedescofona, durante l'età adulta ha raggiunto una buona competenza di italiano. Nel suo ritratto (Figura 17), italiano e tedesco, dalle gambe, si estendono verso le braccia, i cui colori rappresentano un'apertura, in senso generale, verso le lingue straniere.

Figura 17.

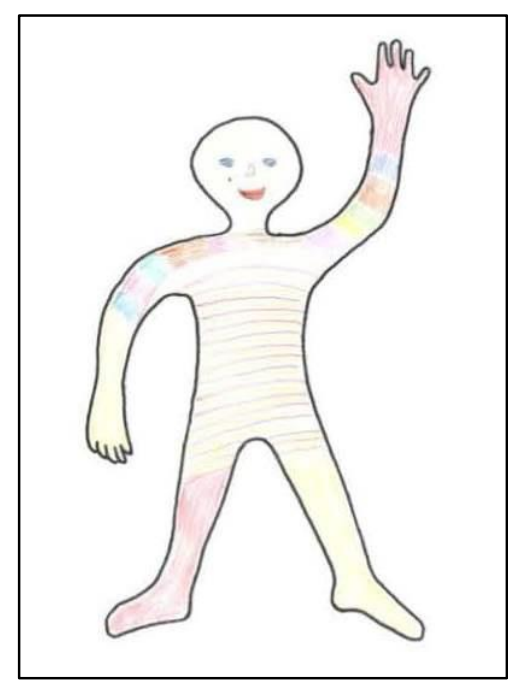


Le domande riguardo il proprio rapporto con l'italiano inducono diversi informatori a tematizzare questioni percepite come particolarmente problematiche nel contesto altoatesino.

Uno dei temi toccati è, ad esempio, il problema dell'identità e del senso di appartenenza ${ }^{23}$. L'informatrice An.st1, pur provenendo da un ambiente prevalentemente tedescofono, racconta di aver sempre cercato il contatto con persone italiane, malgrado le iniziali difficoltà di comunicazione. Nonostante lo stretto rapporto con la lingua e la sua autopercezione come italiana, tuttavia, l'informatrice si trova spesso a fronteggiare i tentativi di delegittimazione operati da interlocutori esterni nei confronti della sua identità.

Probabilmente se parlo dei temi più emotivi o emozionali o che mi sono sul cuore parlo italiano [...] è sempre stata una lingua quando parlavo di emozioni che usavo più per dire cose mie, quando parlavo di emozioni così, perché per me (..) cioè io mi sento anche tanto italiana, anche se so che spesso dagli italiani non vengo riconosciuta come italiana, che è una cosa che mi dà molto fastidio, quando sono all'estero e la gente, anche la gente non italiana ma tutta la gente mi vuole sempre tipo label [=etichettare] come austriaca o tedesca, perché io non mi sento tedesca, quindi per me è una roba che mi dà superfastidio. Se qualcuno vuole che lo odi basta che mi dica che sono austriaca [ride], è una cosa che spesso mi succede viaggiando, che continuamente la gente mi vuole togliere la identità italiana che ho, io ce l'ho. $\mathrm{Da}$ una parte ho un rapporto molto stretto con l'italiano, dall'altra parte è anche qualcosa alla quale magari vorrei più appartenere, e vedo che la gente spesso non vuole che io - o non - non mi considera italiana, anche se io mi sento italiana.

La situazione descritta viene rappresentata anche nel ritratto linguistico dell'informatrice (Figura 18): l'italiano (in verde), occupa la parte del cuore e si mischia con il dialetto nella pancia; il viso, invece, rappresenta ciò che vedono gli esterni, ovvero un'identità tedesca (giallo per l'Hochdeutsch e arancione per il dialetto). Il verde degli occhi, ovvero l'identità italiana, viene riconosciuto solo da pochi.

Figura 18.

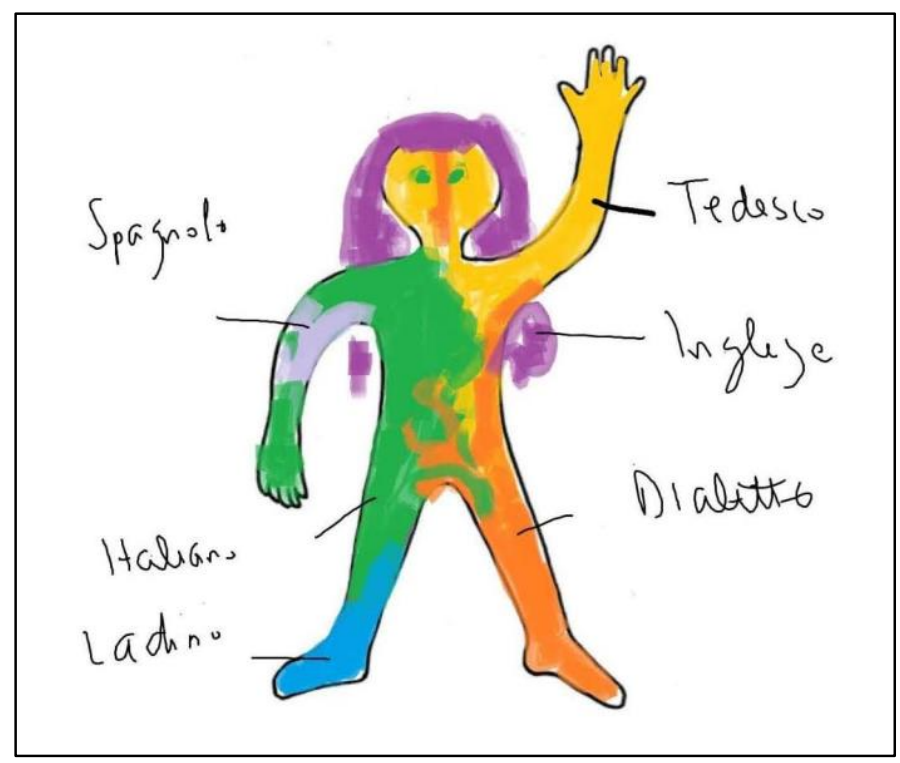

${ }^{23}$ Cfr., tra gli altri, Mioni (1994) e Riehl (2000). 
Nel racconto di An.st1 si coglie il contrasto fra appartenenza («Zugebörigkeit») e non appartenenza («Nichtzugehörigkeił»), identificato da Busch (2013: 19) come uno dei Leitmotiv riscontrabili in molte rappresentazioni del proprio vissuto linguistico: «questo può includere sia il desiderio di identificarsi con un gruppo attraverso la lingua, sia l'esperienza di essere, senza che sia richiesto, assegnati da altri a un particolare gruppo sulla base della lingua».

Anche l'informatore An.st2 problematizza la questione dell'appartenenza nazionale. Nel proprio ritratto linguistico, l'intervistato rappresenta la propria condizione di bilingue attraverso i colori delle bandiere italiana e austriaca (Figura 19), ma al contempo dichiara di non sentirsi davvero appartenente a nessuna delle due nazioni.

An.st2: Siccome qui non siamo veramente tedeschi, ma piuttosto austriaci, per metà austriaci, faccio anche il tricolore austriaco, cioè rosso-bianco-rosso. Praticamente metà austriaco e metà italiano. Penso anche che il cuore - il cuore è un po' entrambi, no?

Penso che entrambe le lingue mi appartengano [...] quindi se qualcuno mi chiedesse se mi considero italiano, perché questa è una delle prime domande che ti fanno se sei un altoatesino di lingua tedesca, in realtà - avrei difficoltà a dire di essere italiano, perché amo la lingua italiana, parlo più - al lavoro quasi sempre italiano, ma come sud- come sudtirolese di lingua tedesca si hanno difficoltà con queste domande.

Si può conoscere bene una lingua, ma non sentirsi comunque parte di quella nazione. Ai mondiali, per esempio, sono sempre dalla parte degli italiani, ma non canto l'inno nazionale. Quando tutti gli altri si alzano per cantare l'inno nazionale, sono in difficoltà, perché per esempio - non conosco nemmeno quello austriaco, l'inno austriaco.

$\mathrm{N}$ : Sente un senso di appartenenza verso l'Austria?

An.st2: Poco, molto poco.

Figura 19.

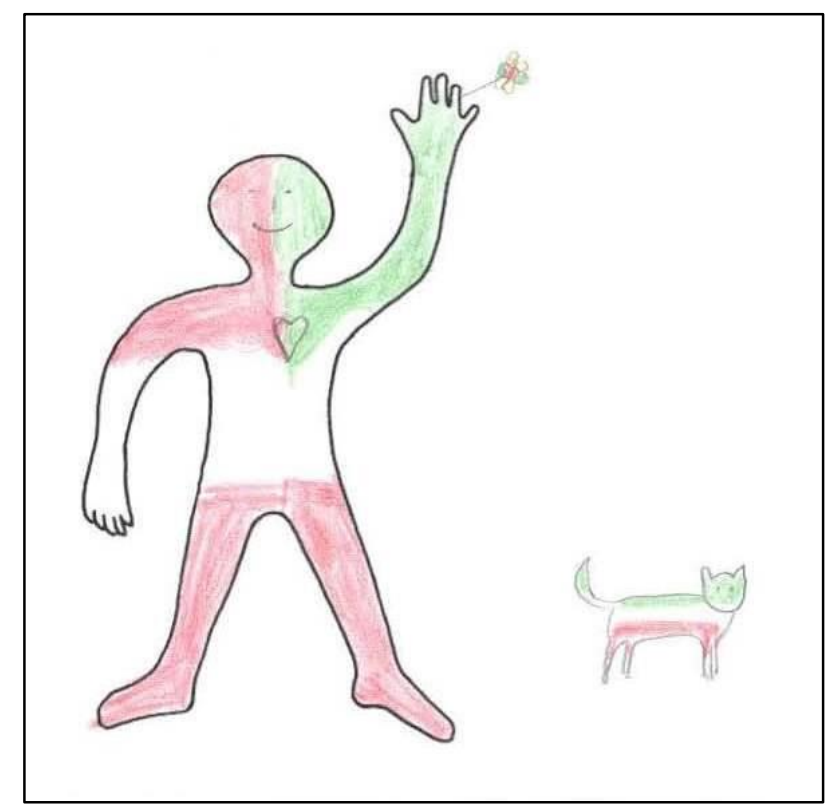


Appaiono rilevanti, a questo proposito, le osservazioni di Riehl (2000: 143):

Nel caso delle minoranze linguistiche, questo [il rapporto con le «identità etniche multiple»] [...] è un po' più problematico [...]. Dopo tutto, la lingua e la cultura del gruppo (di solito limitato a livello regionale) sono diverse dalla lingua e dalla cultura della nazione cui appartengono come cittadini. La lingua della nazione non funge quindi da Dachsprache per la lingua della regione. Ciò significa che l'identità linguistico-culturale regionale e l'identità nazionale possono entrare in conflitto tra loro.

Nel caso delle cosiddette «minoranze di confine», minoranze sorte a seguito delle nuove demarcazioni di confine in Europa nel XX secolo, questo rapporto è in molti casi particolarmente problematico. Infatti, anche se formano una comunità culturale propria, sono parte di un'unità culturale $\mathrm{e}$ linguistica più grande che forma una nazione propria, alla quale non appartengono più.

Ricollegandoci, brevemente, all'impiego del tricolore, è interessante soffermarsi sulla rappresentazione dell'italiano da parte di Pe.st2, apparentemente contraddittoria (Figura 20). L'informatore, che si identifica come sudtirolese tedesco, disegna l'italiano (in giallo) nella testa, per segnalare lo sforzo cognitivo legato all'uso di questa lingua, ma al contempo, percependo «la lingua e la mentalità italiane» come «molto vicine al cuore», rappresenta quest'ultimo con i colori della bandiera italiana.

Figura 20.

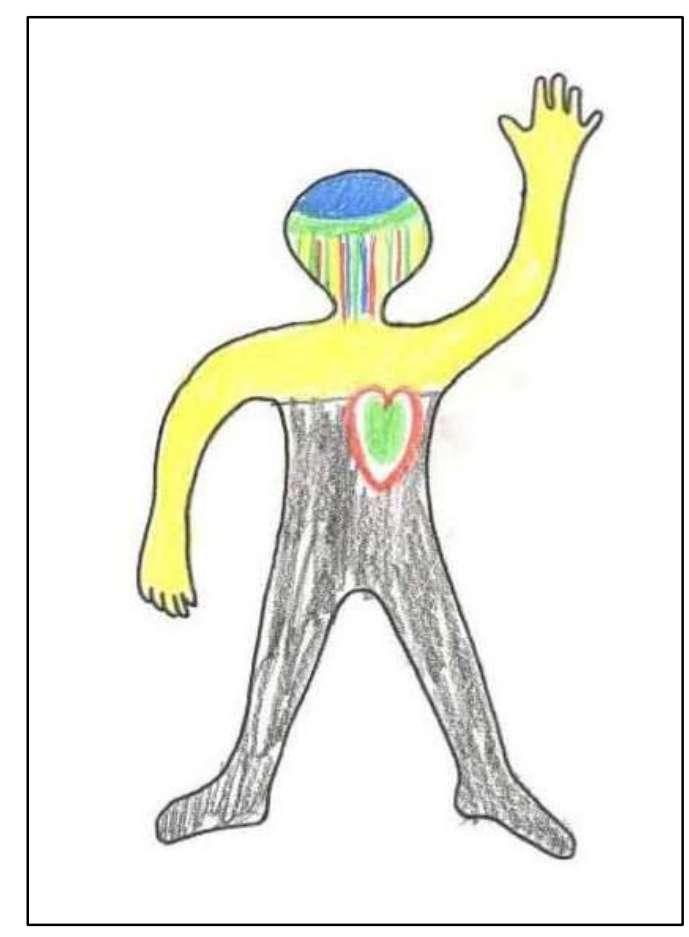

Un'altra tematica che emerge frequentemente riguarda la divisione fra i gruppi linguistici. L'informatore meranese Ga.st1, proveniente da una famiglia italofona ma immerso fin da piccolo in un ambiente tedescofono, parla ad esempio di un «odio razziale» fra i gruppi linguistici. 


\section{$\mathrm{N}$ : Come percepisci l'italiano?}

Ga.st1: Lo sento ugualmente mio, alla fine non è che faccio una distinzione però in Alto Adige comunque avendo - alla fine siamo ancora indietro anche sempre con lingue che (.) «Tu sei italiano», «Tu sei tedesco», con 'sta guerra ancora che purtroppo - vecchia come i sassi (..) Non so in situazioni magari preferisci anche non dire che sei più questo o più quell'altro perciò non saprei non - quasi sei costretto a vergognarti di una parte di te, per dire, dipendente dal contesto che magari ogni tanto giri con italiani che son contro i tedeschi dici «Ok parlo solo italiano» e poi viceversa.

Benché in termini più leggeri, anche l'informatore Aa.st1 esprime il proprio disappunto per la distanza che, nella vita quotidiana, caratterizza il rapporto tra i due gruppi.

C'è anche un po' - non so un po' 'sta troppa paura di (..) sì non c'è abbastanza contatto probabilmente tra o due gruppi linguistici [...] secondo me qua è tutto un po' [...] fuori è tutto "siamo bilingue», l'Alto Adige tutto bello, tutto eh (.) e però poi boh nelle situazioni così, daily life [=vita quotidiana], è tutt'altra cosa, no?

Allo stesso modo, Li.st1 nota la divisione tra italofoni e tedescofoni e la conseguente venuta meno di un'occasione di apprendimento della seconda lingua:

Questo è il problema direi, che non ci sono gruppi di amici misti. Uno poi impara anche il linguaggio giovanile o così con gli amici o anche così in generale (.) nuove parole, nuovo lessico e così e in questo caso (.) per niente - cioè io ho anche amici italiani ma davvero molto pochi.

Diversi informatori dichiarano di ritenere inconcepibile il mancato bilinguismo di parte della popolazione e giudicano come potenzialmente problematica la tendenza dei tedescofoni, spesso menzionata anche in letteratura, a passare all'italiano nell'interazione con l'altro gruppo.

L'informatrice Ev.st2 sottolinea la necessità, a suo avviso, di rivoluzionare il sistema scolastico, portando come esempio l'esperienza negativa della divisione tra i gruppi linguistici riscontrata fin da quando le figlie andavano all'asilo.

$\mathrm{Al}$ piano terra gli italiani e al piano superiore $\mathrm{i}$ tedeschi - una settimana $\mathrm{i}$ tedeschi stavano in questo lato del giardino e la settimana successiva in quello, e gli italiani viceversa. Non si incontravano mai [...] e a scuola lo stesso, al piano di sotto i tedeschi e al piano di sopra gli italiani, gli uni hanno la pausa alle dieci, gli altri alle dieci e mezza; non si vedono quando entrano, uno inizia alle otto e un quarto, l'altro alle otto o giù di lì. C'è solo il rumore che disturba, $\mathrm{ma}-\mathrm{ma}$ non c'era niente insieme, non c'era un progetto insieme (.) lo trovavo terribile.

L'informatrice racconta di essersi scontrata con la stessa divisione cieca quando aveva tentato di introdurre attività condivise tra i due gruppi linguistici nel contesto della propria parrocchia, poi abbandonata proprio per questo motivo; rispetto ai decenni passati, tuttavia, l'informatrice ritiene che, con l'allontanarsi del ricordo dell'oppressione fascista, il rapporto sia in via di miglioramento.

Penso che con la gioventù, con la nuova generazione, ancora migliorerà, penso che questa sia ancora una generazione [che fa fatica a rapportarsi con l'altro gruppo] e penso che venga ancora dalla guerra (.) quindi c'è ancora un 
lungo tempo - non è ancora stato rielaborato completamente il «Eravamo austriaci, eravamo tedeschi e gli italiani ci hanno, diciamo, invaso» ${ }^{4}[\ldots]$ penso che siano successe tante cose che sono semplicemente andate avanti per generazioni, ma che ora si fermeranno nelle prossime.

\section{Conclusione}

Riprendendo un'immagine di Franceschini e Veronesi (2016: 249), possiamo affermare che «le biografie linguistiche producono l'effetto di una lente d'ingrandimento»: sotto i nostri occhi scorrono stralci di storie di vita, idee, desideri, aspirazioni, ricordi e sofferenze; ancora una volta, l'apprendimento linguistico si dimostra legato a doppio filo al vissuto personale e collettivo. Gli autoritratti linguistici rappresentano una sorta di istantanea di tale vissuto, tanto affascinante quanto momentanea, contingente, un singolo fotogramma di un pellicola che continuerà a girare. Per un breve attimo, però, il rullino si ferma: nel processo della riflessione sull'autoritratto linguistico, il soggetto è portato a fare un passo indietro, guardare sotto una luce inedita ciò che ha davanti agli occhi quotidianamente, e, non di rado, scoprirne nuove sfaccettature. Questo è, a nostro avviso, il grande merito dell'impiego dell'autoritratto linguistico: uno strumento apparentemente più adatto ai più piccoli, ma capace, in realtà, di innescare un'autoanalisi di inaspettato interesse in persone di ogni età.

Concludiamo, infine, con una considerazione di Busch (2013: 17) riguardo l'indagine di tipo biografico:

La ricerca biografica nel campo del plurilinguismo rappresenta un campo in espansione, specialmente in relazione a migrazione, mobilità e apprendimento delle lingue in mondi caratterizzati da eteroglossia e riconfigurazione politica degli spazi. Lo sguardo biografico sul repertorio linguistico non è solamente adatto ad assumere una prospettiva orientata al parlante, bensì pone in rilievo aspetti finora poco considerati, quali, ad esempio, l'influenza delle ideologie linguistiche sul modo in cui i parlanti posizionano sé stessi e gli altri a livello discorsivo, o il ruolo delle emozioni, dell'immaginazione e del desiderio in relazione al repertorio linguistico.

\section{RIFERIMENTI BIBLIOGRAFICI}

Adamzik K., Roos E. (eds.) (2002), Biografie linguistiche / Biographies langagières / Biografias linguisticas / Spracbbiografien, Bulletin vals-asla, Institut de linguistique de l'Université, Neuchâtel, 76: https://docplayer.fr/66416270-Hiver-2002-biografie-linguistichebiographies-langagieres-biografias-linguisticas-sprachbiografien.html.

Anfosso G., Polimeni G., Salvadori E. (a cura di) (2016), Parola di sé. Le autobiografie linguistiche tra teoria e didattica, FrancoAngeli, Milano.

Baur S., Mezzalira G., Pichler W. (2008), La lingua degli altri. Aspetti della politica linguistica e scolastica in Alto Adige-Südtirol dal 1945 ad oggi, FrancoAngeli, Milano.

Berruto G., Cerruti, M. (2014), Manuale di sociolinguistica, UTET, Torino.

${ }^{24}$ Si veda ciò che scrivono Baur, Mezzalira, Pichler (2008) rispetto alla mancata rielaborazione del lutto. 
Blondeau N., Salvadori E. (2020), "Autobiografie linguistiche: per una ricostruzione riflessiva degli itinerari esistenziali e professionali degli insegnanti”, in Salvadori E., Blondeau N., Polimeni G. (a cura di), pp. 11-23.

Busch B. (2006), "Language biographies - approaches to multilingualism in education and linguistic research", in Busch B., Jardine A., Tjoutuku A. (eds.), Language Biographies for multilingual learning, PRAESA Occasional Papers, 24, Cape Town, pp. 5-18: https://www.praesa.org.za/wp-content/uploads/2017/01/Paper24.pdf.

Busch B. (2010a), "Die Macht präbabylonischer Phantasien. Ressourcenorientiertes sprachbiographische Arbeiten", in Zeitscbrift für Literatumvissenschaft und Linguistik LiLi, 40, pp. 58-82:

https://heteroglossia.net/fileadmin/user_upload/busch2010_Lili.pdf.

Busch B. (2010b), “"Wenn ich in der einen Sprache bin, habe ich immer auch die andere im Blick»: Zum Konnex von Politik und Spracherleben”, in de Cillia R., Gruber H., Menz F., Krzyzanowski M. (eds.), Diskurs, Politik, Identität. Discourse, politics, identity: Festschrift für Ruth Wodak, Stauffenburg, Tübingen, pp. 235-244:

https://www.univie.ac.at/ie/sprachmittlung/Busch10_FSWodak.pdf.

Busch B. (2012), “The linguistic repertoire revisited", in Applied Linguistics, 33, 5, pp. 503523.

Busch B. (2013), Mehrsprachigkeit, UTB, Stuttgart.

Busch B. (2018), "Das Sprachenportrait in der Mehrsprachigkeitsforschung", in Osnabrücker Beiträge zur Sprachtheorie, 93, pp. 53-70: http://www.lcm.unige.it/polyphonie/upload/16014694270.pdf.

Busch B. (2021), "The body image: taking an evaluative stance towards semiotic resources", in International Journal of Multilingualism, pp. 1-16: https://www.tandfonline.com/doi/epub/10.1080/14790718.2021.1898618?need Access=true.

Canobbio S. (2005), “Autobiografie sociolinguistiche e vicende del territorio”, in Bollettino Linguistico Campano, 7-8, Liguori, Napoli, pp. 73-90.

Cardano M. (2003), Tecniche di ricerca qualitativa. Percorsi di ricerca nelle scienze sociali, Carocci, Roma.

Castellotti V., Moore, D. (2009), "Dessins d'enfants et constructions plurilingues. Territoires imagés et parcours imaginés", in Molinié M. (ed.), pp. 45-85.

Cavagnoli S. (2014), "L'autobiografia linguistica a scuola tra plurilinguismo e affettività", in Landolfi L. (a cura di), pp. 179-188.

Cognigni E. (2014), "Le autobiografie linguistiche a scuola tra plurilinguismo e affettività: dal ritratto al racconto delle lingue", in Landolfi L. (a cura di), pp. 189-200.

Cognigni E. (2020), "Le autobiografie linguistiche tra ricerca e formazione: approcci e metodi di analisi", in Salvadori E., Blondeau N., Polimeni G. (a cura di), pp. 178190.

Corti L. (2012), “Autobiografie linguistiche: un'esperienza condotta con apprendenti l'italiano L2 sinofoni”, Italiano LinguaDue, 4, 1, pp. 448-470:

https:// riviste.unimi.it/index.php/promoitals/article/view/2292.

Dal Negro S. (2012), "Breve introduzione ai metodi di ricerca in sociolinguistica", in Dal Negro S., Provenzano C. (a cura di), Un anno in Lingua 2 / Zweitsprachjahr / N ann te L2 y L3. Strumenti e metodi per la ricerca, Junior, Bergamo, pp. 23-41.

Dell'Aquila V., Iannàccaro G. (2000), “Alla ricerca della Comunità Linguistica: spunti dal concetto di «lingua madre»”, in Marcato G. (a cura di), Isole linguistiche? Per un'analisi dei sistemi in contatto. Atti del Convegno, Sappada/Plodn, Belluno, 1-4 luglio 1999, CLUEP, Padova, pp. 361-371. 
Ellis R. (2001), "The metaphorical constructions of second language learners", in Breen M. P. (ed.), Learner Contributions to Language Learning. New Directions in Research, Routledge, London, pp. 65-85.

Fix U. (2003), "Identität durch Sprache - eine nachträgliche Konstruktion?", in Janich N., Thim-Mabrey C. (eds.), Sprachidentität - Identität durch Sprache, Narr, Tübingen, pp. 107-123.

Franceschini R. (2001), "Sprachbiographien randständiger Sprecher”, in Franceschini R. (ed.), Biographie und Interkulturalität: Diskurs und Lebenspraxis, Stauffenburg, Tübingen, pp. 111-125.

Franceschini R. (2002), "Sprachbiographien: Erzählungen über Mehrsprachigkeit und deren Erkenntnisinteresse für die Spracherwerbsforschung und die Neurobiologie der Mehrsprachigkeit", in Adamzik K., Roos E., (eds.), pp. 19-33.

Franceschini R. (2003), "Unfocussed Language Acquisition? The Presentation of Linguistic Situations in Biographical Narration", in Forum Qualitative Sorialforschung, 4, 3, pp. 29-49:

https://www.researchgate.net/publication/253457350_Unfocussed_Language_A cquisition_The_Presentation_of_Linguistic_Situations_in_Biographical_Narratio n.

Franceschini R. (2004), "Sprachbiographien: das Basel-Prag-Projekt (BPP) und einige mögliche Generalisierungen bezüglich Emotion und Spracherwerb", in Franceschini R., Miecznikowski J. (eds.), pp. 121-145.

Franceschini R., Miecznikowski J. (eds.) (2004), Leben mit mehreren Sprachen. Vivre avec plusieurs langues. Sprachbiographien. Biographies langagières, Peter Lang, Bern.

Franceschini R., Miecznikowski J. (2004), "«Wie bin ich zu meinen verschiedenen Sprachen gekommen?» Ein Vorwort”, in Franceschini R., Miecznikowski J. (eds.), VII-XV.

Franceschini R., Veronesi D. (2016), “Lernerbiographische Perspektiven”, in BurwitzMelzer E., Melhorn G., Riemer C., Bausch K.-R., Krumm H. (eds.), Handbuch Fremdsprachenunterricht, 6, Auflage, Narr Franke Attempto, Tübingen, pp. 247-249.

Groppaldi A. (2010), "L'autobiografia linguistica nell'insegnamento/apprendimento dell'italiano L2/LS", in Italiano LinguaDue, n. 1. 2010, pp. 89-103:

http:/ / riviste.unimi.it/index.php/promoitals/article/view/633.

Guerrero A. L. (2011), "Narrative as resource for the display of self and identity: The narrative construction of an oppositional identity", in Colombian Applied Linguistics Journal, 13, 2, pp. 88-99:

http:/ / www.scielo.org.co/scielo.php?script=sci_arttext\&pid=S012346412011000200007.

Halbwachs M. (1997 [1950]), La mémoire collective, Éditions Albin Michel, Paris.

$\mathrm{Hu}$ A. (2006), "Mehrsprachigkeit und Mehrkulturalität in autobiographischer Perspektive", in FL $u L$, 35, pp. 183-200: https://orbilu.uni.lu/handle/10993/10220.

Iannàccaro G. (2000), "Per una semantica più puntuale del concetto di «dato linguistico»: un tentativo di sistematizzazione epistemologica", in Quaderni di Semantica, XXI, pp. 51-79.

Iannàccaro G. (2002), "La percezione del cambio linguistico nel parlante”, in Cini M., Regis R. (a cura di), Che cosa ne pensa oggi Chiaffredo Roux? Percorsi della dialettologia percerionale all'alba del nuovo millennio. Atti del Convegno Internazionale, Bardonecchia, 25-27 maggio 2000, Edizioni Dell'Orso, Alessandria, pp. 81-108.

Iannàccaro G., Dell'Aquila V. (2006), Survey Ladins: Usi linguistici nelle Valli Ladine, Regione Autonoma Trentino-Alto Adige, Trento: 
https://www.academia.edu/1635334/SURVEY_LADINS._USI_LINGUISTICI _NELLE_VALLI_LADINE

Kalaja P., Melo-Pfeifer S. (eds.) (2019), Visualising Multilingual Lives: More Than Words, Multilingual Matters, Clevedon, UK.

Landolfi L. (a cura di) (2014), Crossroads. Languages in (e)motion, Università degli studi di Napoli "L'Orientale", Napoli:

https://iris.unive.it/retrieve/handle/10278/3678376/79655/emotions\%20landol fi.pdf.

Lupica Spagnolo M. (2015), Biografie linguistiche e ristrutturazione dei repertori tra Alto Adige e Balcani, Tesi di Dottorato di ricerca, Università di Pavia.

Mioni A. M. (1990), "Bilinguismo intra- e intercomunitario in Alto Adige / Südtirol: considerazioni sociolinguistiche", in Lanthaler F. (ed.), Mehr als eine Sprache. Zu einer Sprachstrategie in Südtirol - Più di una lingua. Per un progetto linguistico in Alto Adige, Alpha \& Beta, Merano, pp. 13-36.

Mioni A. M. (1994), "Vivere senza dialetto?", in Lanthaler F. (ed.), Dialekt und Mehrsprachigkeit | Dialetto e plurilinguismo. Beiträge eines internationalen Symposiums. Atti di un simposio internationale, Alpha \& Beta, Merano, pp. 27-47.

Molinié M. (ed.) (2009), Le dessin réflexif : Elément pour une herméneutique du sujet plurilingue, Centre de Recherche Textes et Francophonies, Université de Cergy-Pontoise. https:/ / hal-univ-paris3.archives-ouvertes.fr/hal-01475642/document.

Nekvapil J. (2003), "Language biographies and the analysis of language situations: on the life of the German community in the Czech Republic", in International Journal of the sociology of language, Mouton de Gruyter, Berlin-New York, 162, pp. 63-83:

https:/ /www.researchgate.net/publication/240751056_Language_biographies_an d_the_analysis_of_language_situations_On_the_life_of_the_German_communit y_in_the_Czech_Republic

Pavlenko A. (2007), "Autobiographic Narratives as Data”, in Applied Linguistics, 28, 2, pp. 163-188.

Riehl C. M. (2000), "Nationale und regionale Identität: Das Beispiel der deutschsprachigen Minderheit in Südtirol", in Haslinger P. (ed.), Regionale und nationale Identitäten. Wechselwirkungen und Spannungsfelder im Zeitalter moderner Staatlichkeit, Ergon Verlag, Baden-Baden, pp. 143-153:

https://www.daf.uni-

muenchen.de/personen/professoren/riehl/riehl_publ/papers/cr2000nationale.p df.

Salvadori E., Blondeau N., Polimeni G. (a cura di) (2020), Lingue maestre. Autobiografia linguistica e autoformazione dei docenti di L1 e L2, sezione monografica di Italiano LinguaDue, 12, 2:

https://riviste.unimi.it/index.php/promoitals/article/view/14983.

Telmon T. (2006), "Gli studenti si confessano: considerazioni sulle autobiografie sociolinguistiche", in Marcato G. (a cura di), Giovani, lingue e dialetti. Atti del Convegno, Sappada/Plodn, Belluno, 29 giugno-3 luglio 2005, Unipress, Padova, pp. 221-229.

Thamin N., Simon, D.-L. (2011), "Réflexions épistémologiques sur la notion de «biographies langagières»", in Huver E., Molinié M. (eds.), Praticiens-chercheurs à l'écoute du sujet plurilingue. Réflexivité et interaction biographique en sociolinguistique et en didactique, Carnets d'Atelier de Sociolinguistique, 4, L'Harmattan, Paris, pp. 15-33: https://www.academia.edu/25996707/R\%C3\%A9flexions_\%C3\%A9pist\%C3\% A9mologiques_sur_la_notion_de_biographies_langagi\%C3\%A8res_.

Tophinke D. (2002), "Lebensgeschichte und Sprache. Zum Konzept der Sprachbiografie aus linguistischer Sicht”, in Adamzik K., Roos E. (eds.), pp. 1-14. 
Veronesi D. (2008), "Rappresentazioni di lingue e di percorsi di apprendimento: la metafora nelle biografie linguistiche di parlanti in Alto Adige-Südtirol", in Casadio C. (a cura di), Vie della metafora: linguistica, filosofia, psicologia, Prime Vie, Chieti, pp. 120-145.

Veronesi D. (2009), “«Due lingue che sono entrambe mie»: biografie di parlanti bilingui e monolingui in un territorio di confine", in Riccioni I. (ed.), Multiculturalismi a confronto. Chiapas, Catalogna, Amazzonia peruviana, Alto Adige-Südtirol: la funzione delle minoranze nel mondo globalizzato. Atti del seminario, Bressanone, 20 maggio 2009, Bozen Bolzano University Press, Bolzano, pp. 39-56:

https://www.researchgate.net/publication/285200505_Due_lingue_che_sono_en trambe_mie_biografie_di_parlanti_\%27bilingui $\% 27$ _e_\%27monolingui $\% 27$ in_u n_territorio_di_confine.

Veronesi D. (2019), “«But you're gonna ask me questions, right?» Interactional frame and «for-the-record» orientation in language biography interviews", in Roulston K. (ed.), Interactional Studies of Qualitative Research Interviews, John Benjamins, Amsterdam -Philadelphia, pp. 181-200:

https://www.researchgate.net/publication/309212694_But_you\%27re_gonna_as k_me_questions_right_Interactional_frame_and_for-therecord_orientation_in_language_biography_interviews.

Vietti A. (2008), "Scelta di codice in contesti comunicativi incerti. Il caso delle richieste di indicazioni stradali in Alto Adige/Südtirol”, in Vox Romanica, 67, pp. 34-56.

\section{Sitografia}

Heteroglossia.net, Sprachporträt / Language portrait.

https://heteroglossia.net/Sprachportraet.123.0.html. 